\title{
Dynamics of Pulsating, Erupting, and Creeping Solitons in the Cubic- Quintic Complex Ginzburg-Landau Equation under the Modulated Field
}

\author{
Woo-Pyo Hong \\ Department of Electronics Engineering, Catholic University of Daegu Hayang, Gyongsan, \\ Gyungbuk 712-702, South Korea
}

Reprint requests to Prof. W.-P.H.; E-mail: wphong@cu.ac.kr

Z. Naturforsch. 61a, 525 - 535 (2006); received August 9, 2006

\begin{abstract}
It is shown that the dynamics of the pulsating, erupting, and creeping (PEC) solitons in the onedimensional cubic-quintic complex Ginzburg-Landau equation can be drastically modified in the presence of a modulated field. We first perform the linear instability analysis of continuous-wave (CW) and obtain the gain by the modulational instability (MI). It is found that the CW states applied by the weakly modulated field always transform into fronts for the parameters of the PEC solitons. We then show that, when the modulated field is applied to the pulse-like initial profile, multiple solitons are formed for the parameters of the pulsating and erupting solitons. Furthermore, as the strength of the gain term increases, the multiple pulsating or erupting solitons transform into fixedshape stable solitons. This may be important for a practical use such as to generate multiple stable femtosecond pulses. For the case of creeping soliton parameters, the presence of a modulated field does not generate multiple solitons, however, the initial profile transforms into an irregularly pulsating soliton or evolves into a fixed-shape soliton as the strength of the gain term is increased. - PACS numbers: 42.65.Tg, 03.40.Kf, 05.70.Ln, 47.20.Ky
\end{abstract}

Key words: Pulsating, Erupting, and Creeping Solitons; Modulational Instability; Modulated Field; Fixed-Shape Soliton.

\section{Introduction}

The complex Ginzburg-Landau equation (CGLE) is one of the widely studied nonlinear equations for describing a variety of phenomena in the pattern formation originating from the competition of loss, finitebandwidth gain, nonlinearity, and dispersion [1]. The CGLE and its extension makes it possible to model a wide range of dissipative physical systems, such as binary fluid convection [2], electro-convection in nematic liquid crystals [3], patterns near electrodes in gas discharges [4], and oscillatory chemical reactions [5]. The solitons in such dissipative systems are quite different from those in conservative systems; in fact they are far from equilibrium and have nontrivial internal energy flows from an external source [6]. In particular, for the cubic-quintic extension of the CGLE (cqCGLE), which can model the physical phenomena occurring in passively mode-locked lasers [7], the pulsating erupting and creeping (PEC) solitons have been obtained [8] and experimentally verified $[9,10]$; they strongly depend on an energy supply from an external source while the soliton's energy is continuously dis- sipating and redistributing between its parts [9]. The dissipative solitons can take different shapes and also show a variety of periodic pulsations and chaotic explosions. In particular, the dissipative solitons behave like an attractor in the sense that a wide variety of localized initial conditions converge to it [6].

In this paper, we consider the cqCGLE

$$
\begin{aligned}
& \mathrm{i} \psi_{z}+\frac{d}{2} \psi_{\tau \tau}+|\psi|^{2} \psi+v|\psi|^{4} \psi \\
& =\mathrm{i} \delta \psi+\mathrm{i} \beta \psi_{\tau \tau}+\mathrm{i} \varepsilon|\psi|^{2} \psi+\mathrm{i} \mu|\psi|^{4} \psi
\end{aligned}
$$

in the context of the nonlinear optics, where $d, \delta, \varepsilon$, $\beta, v$ and $\mu$ are real constants. The physical meaning of each particular term depends on the dynamical system under consideration. For example, in the mode-locked laser system, $\psi(z, \tau)$ is the normalized amplitude, $z$ is the propagation distance or the cavity round-trip number (treated as a continuous variable), $\tau$ is the retarded time, $d$ is the group-velocity dispersion coefficient with $d= \pm 1$ depending on anomalous $(d=1)$ or normal $(d=-1)$ dispersion, $\delta$ is the linear gain $(\delta>0)$ or loss $(\delta<0)$ coefficient; $\beta$ accounts for spectral filtering or linear parabolic gain $(\beta>0)$ due to an amplifier, 
the $\varepsilon$ term represents the nonlinear gain (which arises, e. g., from saturable absorption), the term with $\mu$ represents, if negative, the saturation of the nonlinear gain, and the one with $v$ coefficients corresponds, also if negative, to the saturation of the nonlinear refractive index $[6,8,9]$.

We expect many interesting dynamical features beyond the PEC solitons to be discovered by performing numerical simulations since the parameter ranges of the cqCGLE and its extended equations are wide. For example, in the context of the cqCGLE with the parameters of the PEC solitons, Tian et al. [11] have recently analyzed the effect of nonlinear gradient terms on the solitons. They have found that the nonlinear gradient terms can eliminate the periodicity ofthe pulsating and erupting solitons and transform them into fixed-shape solitons, while for the creeping soliton, the nonlinear gradient terms can cause it to breathe periodically at different frequencies on one side and to spread rapidly on the other side. The results have shown that even small nonlinear gradient terms can cause dramatic changes in the behavior of the solution, such as causing opposite sides of the breathing solution to breathe at different frequencies. More recently, Song et al. [12], in the context of the cqCGLE with the third-order dispersion, have shown that even small third-order dispersion can dramatically alter the behavior of the solitons such as eliminating the periodicity of the pulsating and creeping solitons, transforming them into fixed-shape solitons, and altering the explosion of the erupting soliton, causing the eruptions to appear asymmetrically or making the erupting soliton to become chaotic for a little larger third-order dispersion. On the other hand, Hong [13] has previously investigated by numerical simulations the effect of the nonlinear gradient terms on the dynamics of the solitons induced by modulational instability (MI). It was shown that small nonlinear gradient terms make the MI-induced solitons travel along the distance and introduce both elastic and inelastic interactions between the solitons.

The purpose of the present work is to study the effect of the weakly modulated field on the dynamics of the PEC solitons in the context of the cqCGLE. We will show that even though the continuous wave $(\mathrm{CW})$ state under MI for the parameters corresponding to the PEC solitons always transforms into a front, the initial sech-type pulse in the presence of the weakly modulated field produces the multiple pulsating solitons which can be evolved into stable fixed-shape solitons by controlling the spectral filtering or linear parabolic gain value $\beta$ due to an amplifier in (1). This may be very useful in generating multiple femtosecond stable pulses in optical fiber systems. We will also show that the modulated field induces the multiple erupting solitons. In addition, we will show that the modulated field affects the nature of the creeping soliton, of which the motion and stability can also be controlled by the gain term $\beta$.

The paper is organized as follows. In Section 2 we calculate the MI gain of the cqCGLE by linear instability analysis and investigate numerically the evolution of initial CW under MI for the parameters of the PEC solitons. Then, we investigate the effects of the modulated field and the gain term $\beta$ on the evolution of a sech-type pulse for the PEC parameters and the stability of the solitons. Our conclusions are followed in Section 3.

\section{Effects of the Modulated Field on the PEC Solitons}

In this section, we numerically investigate the evolutions of the $\mathrm{CW}$ under $\mathrm{MI}$ and the pulse-type initial profile in the presence of the modulated field for the parameters of the PEC solitons. We adopt the widely utilized split-step Fourier method and take the CrankNicholson implicit scheme for time propagation under periodic boundary condition [13-15].

For the sake of completeness, we would like to present in Figs. $1 \mathrm{a}-\mathrm{c}$ the evolutions of the initial pulse $\psi_{0}(\tau)=\operatorname{sech}(\tau)$ for the pulsating and erupting solitons, respectively, and the analytic function $\psi_{0}(\tau)=$ $\tanh (\tau+10)-\tanh (\tau-10)+0.4 \operatorname{sech}(\tau)$ for the creeping soliton, which confirm the characteristics of the PEC solitons as previously found in [8]. The parameters for the PEC solitons are: (i) pulsating soliton: $d=1, \varepsilon=0.66, \delta=-0.1, \beta=0.08, \mu=-0.1, v=$ -0.1 ; (ii) erupting soliton: $d=1, \varepsilon=1.0, \delta=-0.1$, $\beta=0.125, \mu=-0.1, v=-0.1$; (iii) creeping soliton: $d=1, \varepsilon=1.3, \delta=-0.1, \beta=0.101, \mu=-0.3$, $v=-0.101[8,9]$. The normalized energy as afunction of the distance, defined as $R_{\mathcal{E}}(z) \equiv \mathcal{E}(z) / \mathcal{E}(0)$, where $\mathcal{E}(z)=\int_{-\infty}^{+\infty}|\psi(z, \tau)|^{2} \mathrm{~d} \tau$, is calculated in Fig. 1d for the parameters of the PEC solitons. The periodic oscillations of the pulsating (solid line) and creeping (dashed line) solitons and the rapid variation of the energy for the case of erupting (dot-dashed line) soliton are clearly demonstrated. It has been numerically confirmed, even not shown here, for a wide class of initial localized profiles, that the PEC solitons work as attrac- 

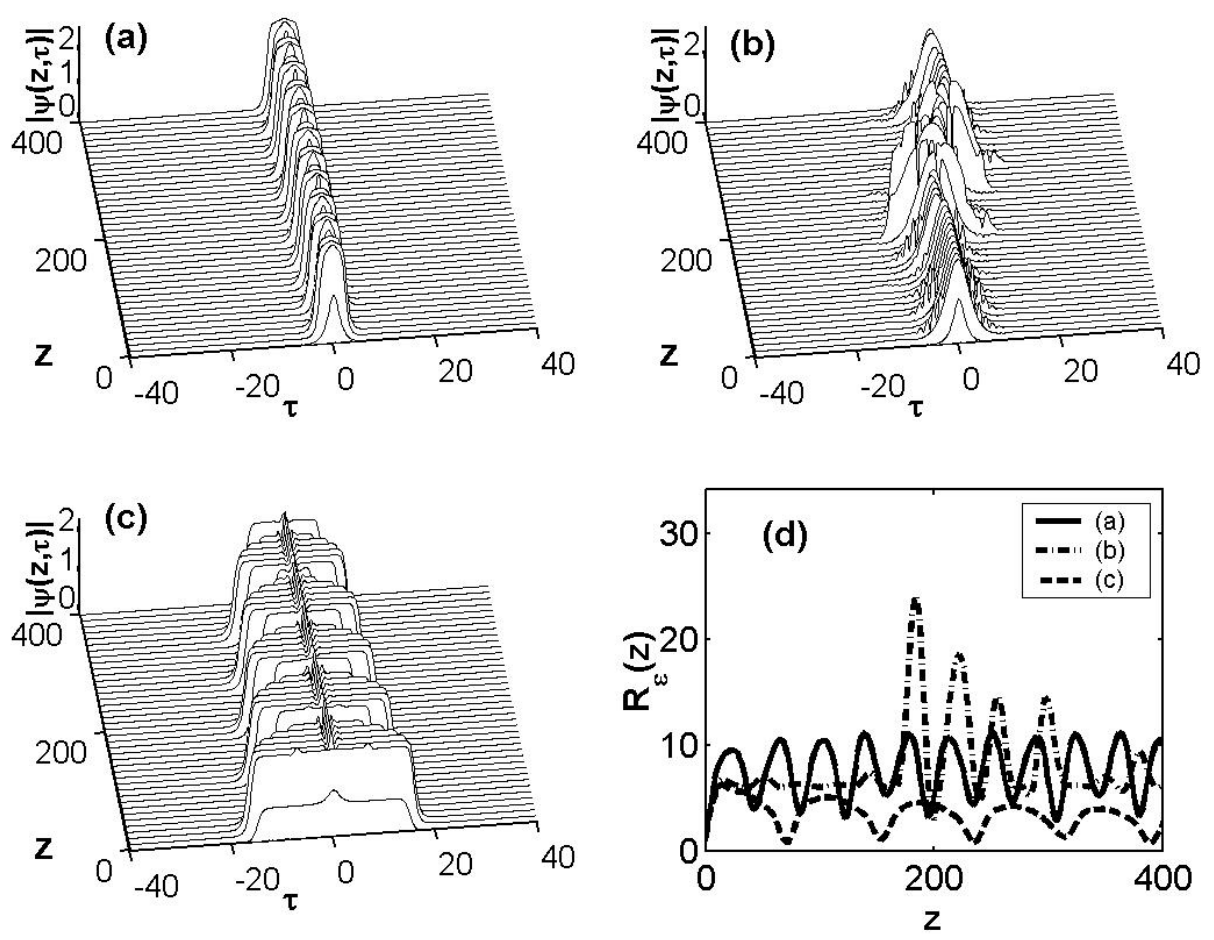

Fig. 1. Evolutions of the (a) pulsating, (b) erupting, and (c) creeping solitons, respectively, confirming the results in [8]. (d) The normalized energies $R_{\varepsilon}(z)$ as functions of the distance for the pulsating (solid line), erupting (dot-dashed line) and creeping (dashed line) solitons, respectively.

tors, i. e., as long as the initial profile remains as a single pulse, the evolution of the pulse belongs to one of the PEC solitons. Thus, for the following analysis, we fix the initial profile as sech function for the pulsating and erupting solitons without losing any generality.

\subsection{Dynamics of Continuous-Wave under Modulational Instability}

At first, we analytically calculate the MI gain function of (1) from the linear instability of CW. We introduce a weakly perturbed field $\eta(z, t)$ such that $\psi(z, \tau)=[A+\eta(z, \tau)] \exp \left[\mathrm{i} \phi_{\mathrm{NL}}(z)\right]$, where the nonlinear phase shift $\phi_{\mathrm{NL}}(z)=z A^{2}+z v A^{4}+\mathrm{i}\left(-z \delta-z \varepsilon A^{2}-\right.$ $\left.z \mu A^{4}\right)$ with the complex field under $|\eta(z, \tau)| \ll A[13-$ 15]. After substituting the perturbed field into (1) and collecting the linear terms in $\eta$, we obtain the linearized equation for the perturbed field as

$$
\begin{aligned}
\mathrm{i} \eta_{z} & +\left[(v-\mathrm{i} \mu) A^{4}+\mathrm{i} \delta\right] \eta+\left(\frac{1}{2} d-\mathrm{i} \beta\right) \eta_{\tau \tau} \\
+ & {\left[(2 v-2 \mathrm{i} \mu) A^{4}+(1-\mathrm{i} \varepsilon) A^{2}\right] \eta^{*}=0 }
\end{aligned}
$$

where $*$ denotes complex conjugate. We assume a gen- eral solution of the form

$$
\begin{aligned}
& \eta(z, \tau)=U \exp [\mathrm{i}(K z-\Omega \tau)] \\
& +V \exp [-\mathrm{i}(K z-\Omega \tau)],
\end{aligned}
$$

where $K$ and $\Omega$ represent the wave number and the frequency of modulation [14], respectively. Inserting (3) into (2), requiring the determinant to be zero, and solving it for the wave number $K$, we obtain the dispersion relation

$$
K=\frac{\mathrm{i}}{2} \sqrt{K_{\mathrm{r}}+\mathrm{i} K_{\mathrm{i}}}
$$

where

$$
\begin{aligned}
K_{\mathrm{r}}= & \left(4 \beta^{2}-d^{2}\right) \Omega^{4}+\left[(4 d v-8 \beta \mu) A^{4}+8 \delta \beta\right] \Omega^{2} \\
& +\left(12 v^{2}-12 \mu^{2}\right) A^{8}+(-16 \mu \varepsilon+16 v) A^{6} \\
& +\left(-8 \mu \delta+4-4 \varepsilon^{2}\right) A^{4}+4 \delta^{2} \\
K_{\mathrm{i}}= & 4 d \Omega^{4} \beta+\left[(-4 \mu d-8 \beta v) A^{4}+4 d \delta\right] \Omega^{2} \\
& -24 v A^{8} \mu+(-16 \mu-16 \varepsilon v) A^{6} \\
& +(-8 \delta v-8 \varepsilon) A^{4} .
\end{aligned}
$$

Expressing (4) in polar coordinate, we obtain

$$
K=\frac{\mathrm{i}}{2}\left[K_{\mathrm{r}}^{2}+K_{\mathrm{i}}^{2}\right]^{1 / 4}\left[\cos \left(\frac{\theta}{2}\right)+\mathrm{i} \sin \left(\frac{\theta}{2}\right)\right],
$$


(a)

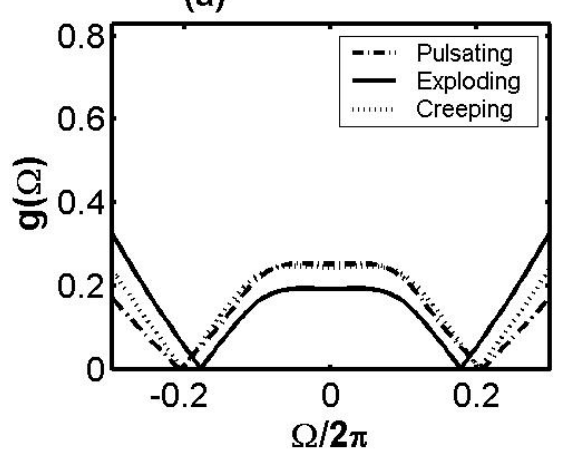

(c) Exploding

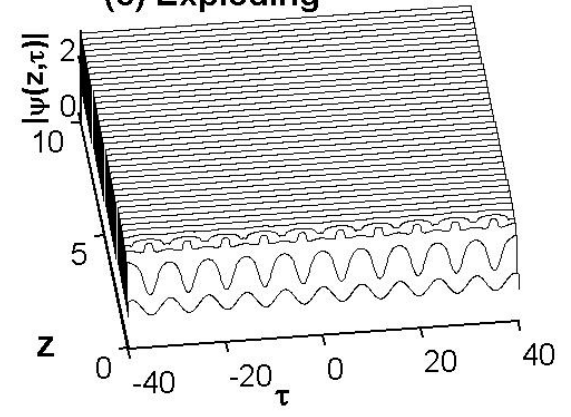

(b) Pulsating

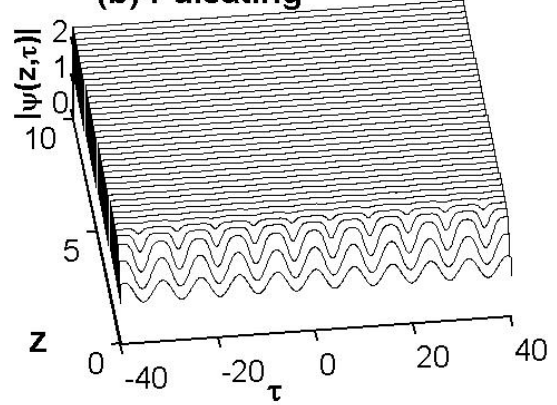

(d) Creeping

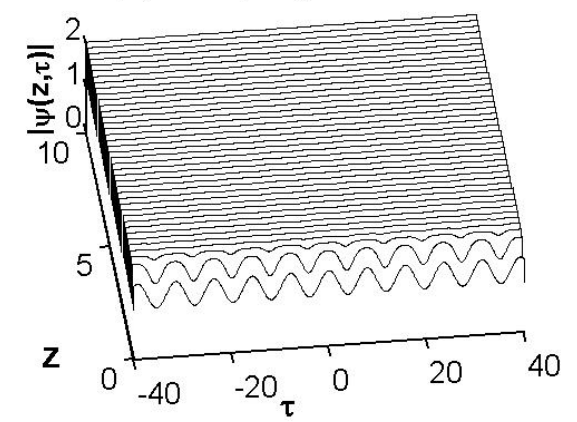

Fig. 2. (a) Plots of the MI spectra as a function of the modulation frequency for the parameters of the pulsating, exploding, and creeping solitons, respectively, where the amplitude is taken as $A=1$. Note the nonzero gain even at $\Omega=0$ for the parameters of the PEC solitons. The MI gain increases as $\Omega$ increases. The space-time evolutions of the $\mathrm{CW}$ under the MI for the parameters of (b) pulsating, (c) exploding, and (d) creeping solitons, respectively, are plotted. Only fronts are generated from the CWs under the MI. where $\cos (\theta)=K_{\mathrm{r}} / \sqrt{K_{\mathrm{r}}^{2}+K_{\mathrm{i}}^{2}}$. The steady-state solution becomes unstable whenever $K$ has an imaginary part since the perturbation grows exponentially with the intensity given by the MI gain defined as $g(\Omega) \equiv$ $2 \operatorname{Im}(K)[14]$ :

$$
g(\Omega)=\frac{1}{2}\left[2 \sqrt{K_{\mathrm{r}}^{2}+K_{\mathrm{i}}^{2}}+2 K_{\mathrm{r}}\right]^{1 / 2} .
$$

The characteristic MI gain spectra are plotted in Fig. 2a as a function of the modulation frequency for the parameters of the PEC solitons [8,9]. It is interesting to note for all the PEC parameters that there exist flat gains for $|\Omega / 2 \pi| \leq 0.1$ due to the presence of the constant parameters terms in (5). Previously, Soto-Crespo et al. [16] have shown that for all higheramplitude $\mathrm{CW}$ solutions $(A \gg 0.1)$ there are regions of stability and regions where the CW solutions are modulationally unstable, while low-amplitude CW solutions are always modulationally unstable. In this context, one expects that the initial high-amplitude $\mathrm{CW}$ under MI transforms into the PEC solitons for the parameters belonging to the existence of a stable single pulse region [16]. However, on the contrary to the expectation, our numerical simulations in Figs. $2 b-d$ show that for the high-amplitude $\mathrm{CW}$, applied with the weakly modulated field in the form of $\psi_{\text {init }}(\tau)=A+$ $\varepsilon_{\mathrm{m}} \cos \left(\Omega_{\mathrm{m}} \tau\right)$ where $A=1, \varepsilon_{\mathrm{m}}=0.2$, and $\Omega_{\mathrm{m}}=0.4$, all the profiles quickly transform into fronts. In fact, similar results have been numerically confirmed for the parameters in the ranges $0.01 \leq \varepsilon_{\mathrm{m}} \leq 0.8,0.4 \leq \Omega_{\mathrm{m}} \leq$ 5.0, and $1 \leq A \leq 10$. This apparent contradiction can be understood from the fact that there is an indirect relation between the stability of the soliton solution and the MI of the higher-amplitude $\mathrm{CW}$ solution, but there is no one-to-one correspondence between them as discussed in [16]. In other words, for parameters of the PEC solitons, even though a pulse-type initial profile can evolve into the PEC solitons, the modulationally unstable $\mathrm{CW}$ does not always transform into a stable soliton.

In the rest of the paper, we will investigate how the dynamical behavior of a Pulse-type initial profile under MI can be modified, which to the author's knowledge has not been considered in previous works $[8,9,16]$.

\subsection{Effect of the Modulated Field on the Pulsating Soliton}

At first we calculate the effect of weakly modulated field on the pulsating soliton when the non- $\mathrm{CW}$ initial profile is modulated by

$$
\psi(0, \tau)_{\text {init }}=\psi_{0}(\tau)+\varepsilon_{\mathrm{m}} \cos \left(\Omega_{\mathrm{m}} \tau\right)
$$


(a) $\beta=0.08$

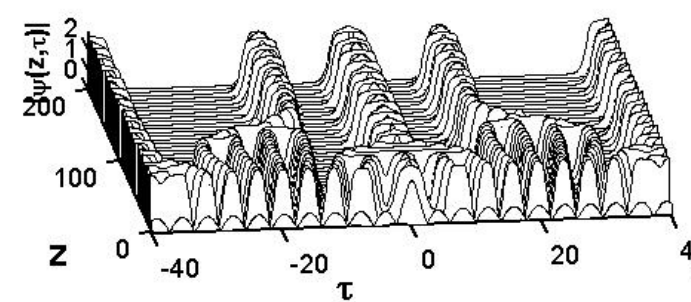

(b) $\beta=0.158^{-40}$
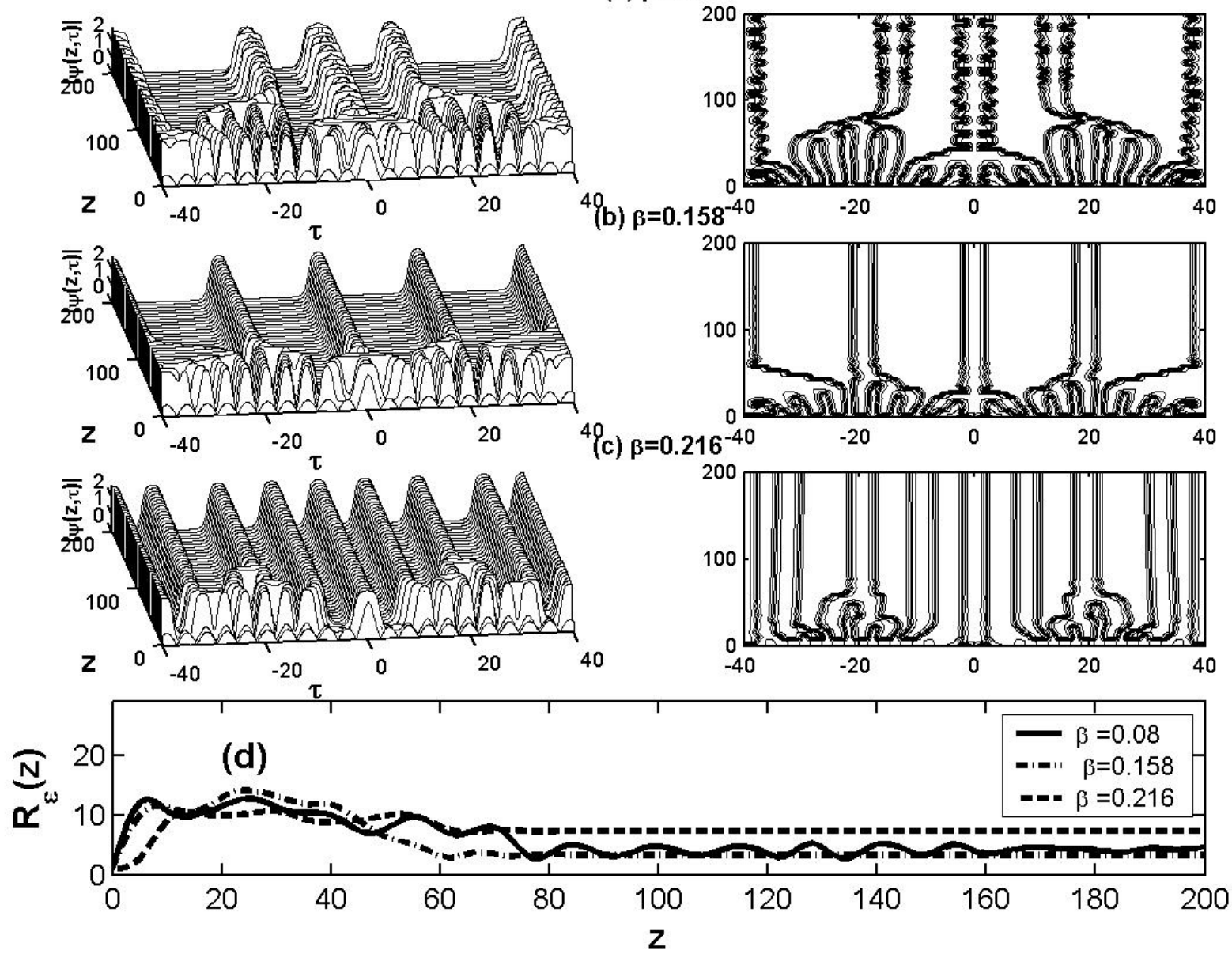

Fig. 3. The space-time evolution and contour plots of the modulus $\psi$ for the parameters of the pulsating soliton with different gain values $\beta$. (a) The appearance of two additional pulsating solitons on the left- and the right-hand side of the initial sech profile and one on the boundaries due to the transient solitons. (b) For $\beta=0.158$, the pulsation of solitons diminishes. (c) For $\beta=0.216$, the transformation of initial profile into multiple fixed-shape solitons after a short transient distance occurs. (d) The evolutions of the normalized energies for (a)-(c), respectively, as functions of the distance $z$. The pulsation in the energy converges to constant values as $\beta$ increases, indicating the stability of the multiple pulsating solitons.

where $\psi_{0}(\tau)=\operatorname{sech}(\tau)$. The same parameters for the modulated field as in Fig. 2 are used in the following numerical simulations. The space-time evolution and contour plots of the modulus $\psi$ are shown in Figs. $3 a-c$ for the pulsating soliton parameters of Fig. 1a and for different $\beta$ values. The presence of the modulated field results in dramatic effects on the dynamics of the pulsating soliton as shown in Fig. 3a: Firstly, we observe the appearance of two additional pulsating solitons on the left- and the right-hand side of the initial sech profile and one on the boundaries, of which the behaviors are overall similar to that of the single pulsating soliton in Figure 1a. Secondly, we find the transient stage during which the pulsating solitons are generated by the cohesion of the transient solitons, indicating the role of the modulated field as the seed for the transient solitons. We also note that for a slight increase of the gain value up to $\beta=0.158$ the pulsations of solitons diminishe as clearly depicted in the contour plot of Figure 3b. However, for $\beta=0.216$ in Fig. 3c, we find the transformation of the initial profile into multiple fixed-shape solitons after a short transient distance. We have performed many numerical simulations with increasing the gain value up to $\beta=0.5$ to find that the stable fixed-shape solitons still appear. In Fig. 3d, we further elaborate the characteristics of 

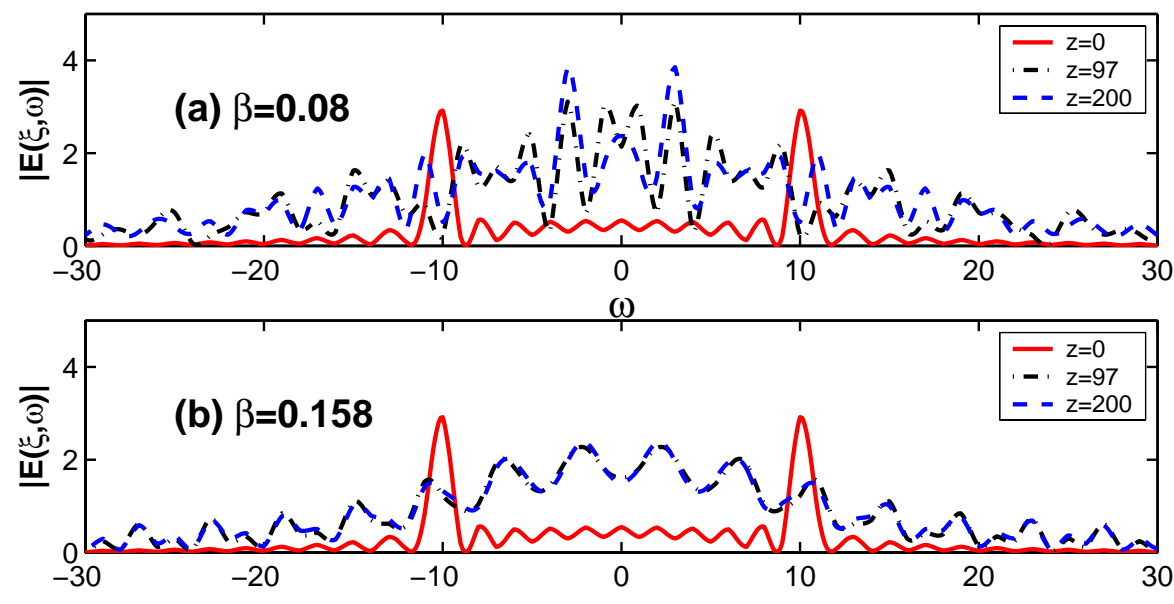

Fig. 4. Plots of the Fourier spectrum amplitudes at different evolution distances of Figures $3 a-c$. (a) Two sech-shaped spectra with the oscillatory wings (solid line), due to the presence of the modulated field, evolve to more complicated symmetric spectra as the distance increases. (b) Almost identical spectrum shapes at $z=97$ and $z=100$, respectively, which confirm the stability of the multiple solitons. (c) Several symmetric spectrum peaks without having any oscillatory wings appear, as the consequence of early stabilization of the multiple solitons.

the solitons by calculating the evolutions of the normalized energies as functions of the distance $z$ which correspond to Figs. $3 \mathrm{a}-\mathrm{c}$, respectively. The solid line clearly demonstrates the periodic pulsation in the energy after the sharp initial peak due to the presence of the cohesion of the transient solitons. Even for a slight increase in $\beta$, the energy oscillation represented by the dot-dashed line converges to a constant value $R_{\varepsilon} \approx 3$, indicating the stability of the multiple pulsating solitons. However, for $\beta=0.216$, the dotted lined shows that the energy oscillation disappears at earlier distance $z \approx 70$ and converges to a constant value $R_{\varepsilon} \approx 8$. These results indicate that the role of gain term $\beta$ is not only to transform the pulsating soliton to the fixed-shape soliton but also to decrease the cohesion distance of the transient solitons.

To better understand the formation of multiple fixedshape solitons by the modulated field, we perform the Fourier transform of the field defined as $\psi(z, \omega)=$ $\int_{-\infty}^{+\infty} \psi(z, \tau) \mathrm{e}^{\mathrm{i} \omega \tau} \mathrm{d} \tau$ and plot the spectrum amplitudes at different evolution distances in Figs. $4 \mathrm{a}-\mathrm{c}$ for the field amplitudes corresponding to Figs. $3 \mathrm{a}-\mathrm{c}$, respectively. It can be seen from Fig. 4a that the evolution starts from two sech-shaped spectra with the oscillatory wings (solid line) due to the presence of the mod- ulated field. Subsequently, while the high frequency component of the multiple solitons spectrum is weakened, there appear symmetric central peaks at $z=97$ (dot-dashed line). Furthermore, the central peak and two side lobes along with the oscillatory wings appear at $z=200$ (dashed line) due to the pulsating nature of the multiple solitons. On the other hand, Fig. $4 \mathrm{~b}$ for the case of $\beta=0.158$ shows almost identical spectrum shapes at $z=97$ and $z=100$, respectively, which confirm the stability of the multiple solitons and demonstrate symmetric spectral broadening over the whole frequency range. Finally, as shown in Fig. $4 c$ for the case of $\beta=0.216$, we find the appearance of several symmetric spectrum peaks without any oscillatory wings, as the consequence of early stabilization of the multiple solitons as shown in Figure 3c. After performing many numerical simulations for the parameters region where the plain pulsating solitons can exist, we conclude that the same evolution and spectrum patterns as shown in Figs. 3 and 4 appear in the presence of increasing $\beta$ values.

In comparison with the role of the nonlinear gradient terms added to the cqCGLE as previously investigated by Tian et al. [11], who eliminated the periodicity of the pulsating soliton and transformed it into 
(a) $\beta=0.125$
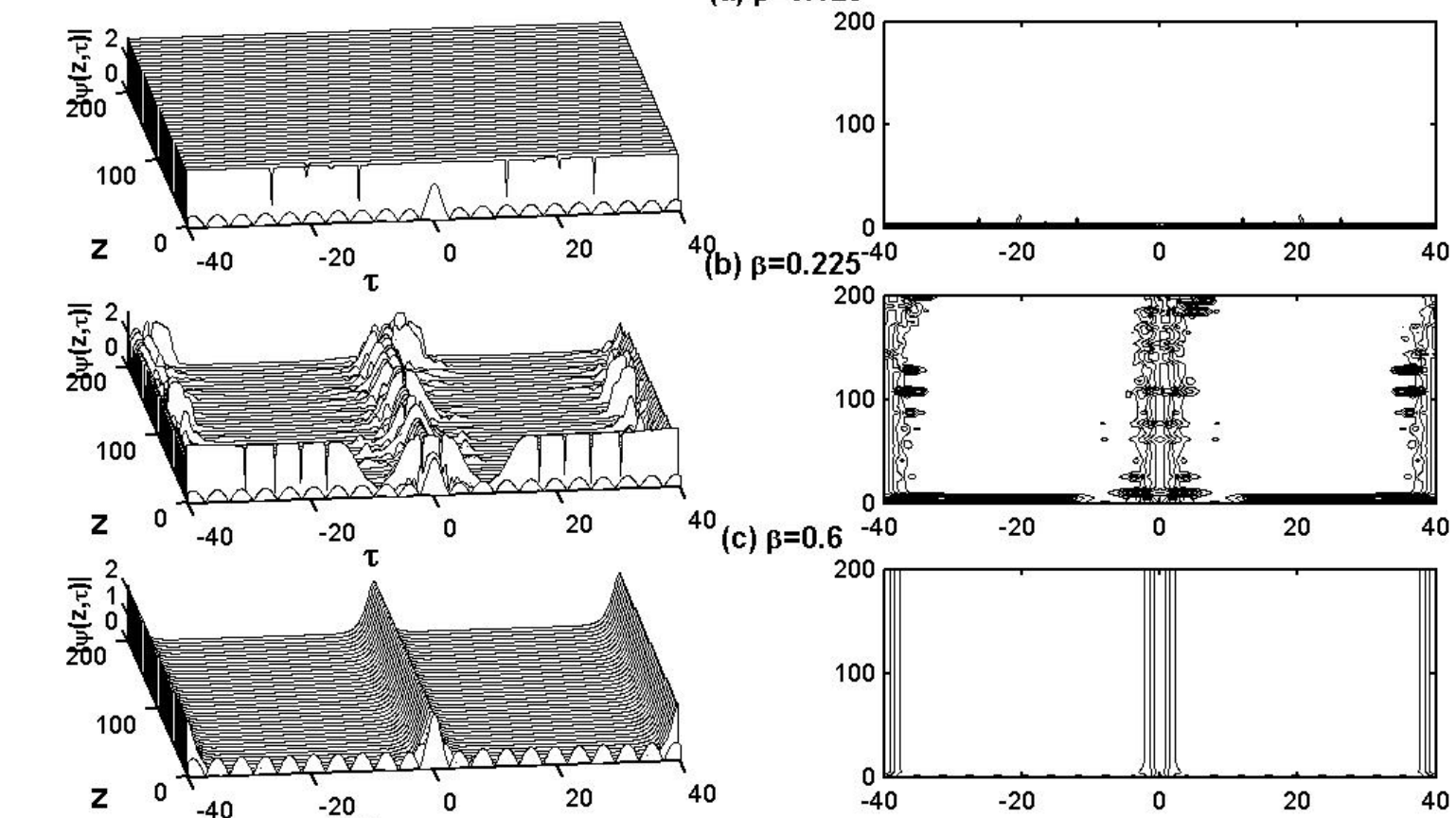

(c) $\beta=0.6$
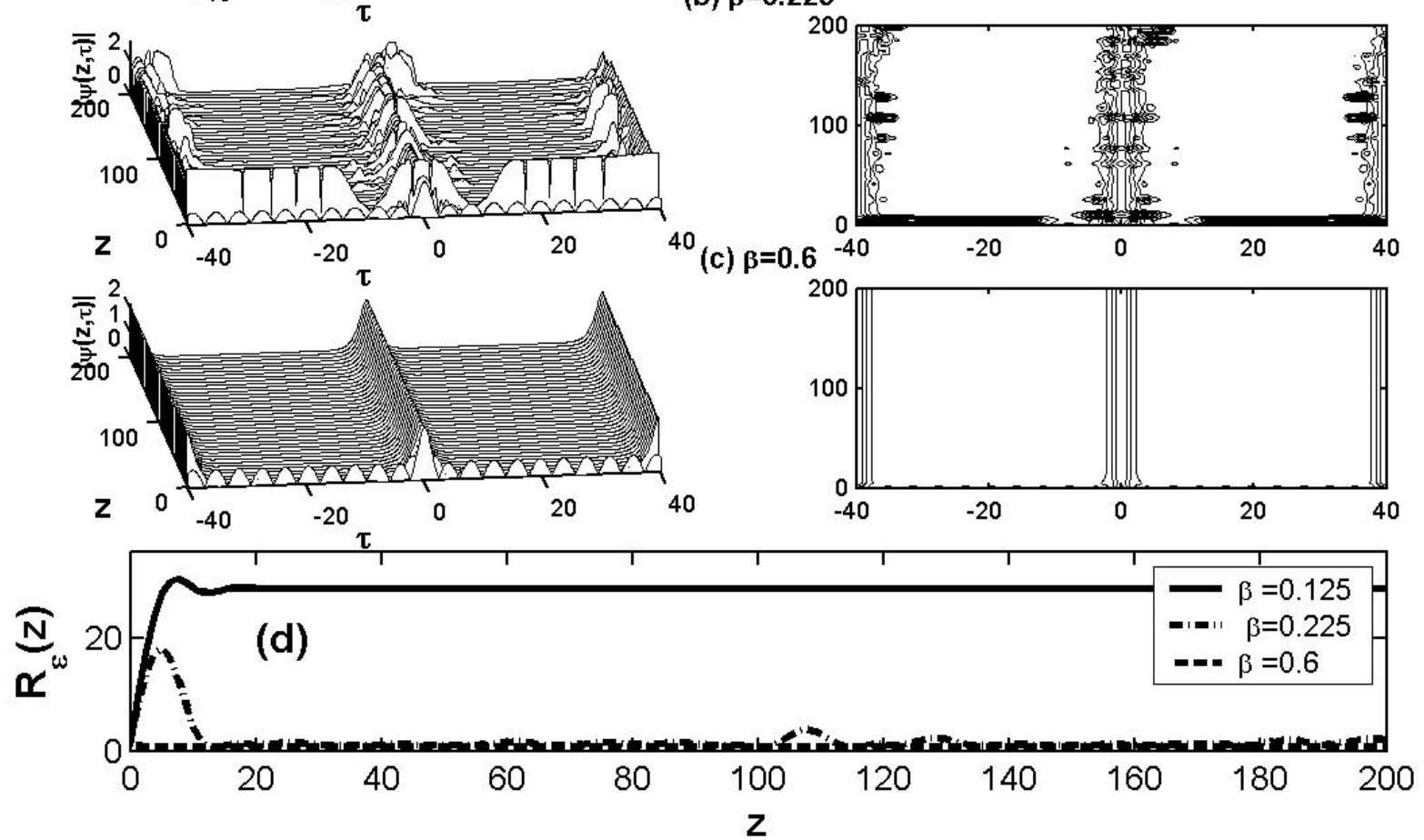

Fig. 5. The space-time evolution and contour plots of the modulus $\psi$ for the parameters of the erupting soliton with different gain values $\beta$. (a) The front formation occurs from the initial sech profile under the modulated field. (b) Two erupting solitons occur from the initial pulse at the center and the decay of the wall-like structure is induced by the modulated field. (c) The initial profile transforms into the two solitons with fixed-shape. (d) The effects of the gain term $\beta$ on the normalized energy evolutions.

a fixed-shape soliton, the fact that the modulated field generates the multiple fixed-shape solitons with stability by simply controlling the $\beta$ term is quite intriguing. In general, the multiple solitons in mode-locked laser systems can be generated by the MI of CW in the parameters regions for allowing stable solitons. However, it was shown by Hong [13] in the context of the extended cqCGLE that the multiple solitons induced by MI interact inelastically along the evolution distance so that it may not be practical to use them as femtosecond pulses for an optical system. Thus, our results in this work may be experimentally more useful and feasible for generating femtosecond pulses in the optical systems modeled by the cqCGLE without resorting to MI.
Finally, we have confirmed the stability of the multiple solitons up to $z=1000$ for various magnitudes of the modulated field and modulation frequencies.

\subsection{Effect of the Modulated Field on the Erupting Soliton}

Next, we investigate the effect of a modulated field on the evolution of the erupting soliton which undergoes an explosive transient stage before returning to its initial state $[8-10,16]$. The space-time evolution and contour plots of the modulus $\psi$ are shown in Figs. $5 \mathrm{a}-\mathrm{c}$ for the erupting soliton parameters of Fig. $1 \mathrm{~b}$ for different $\beta$ values and using the same parameters as above for the modulated field. 

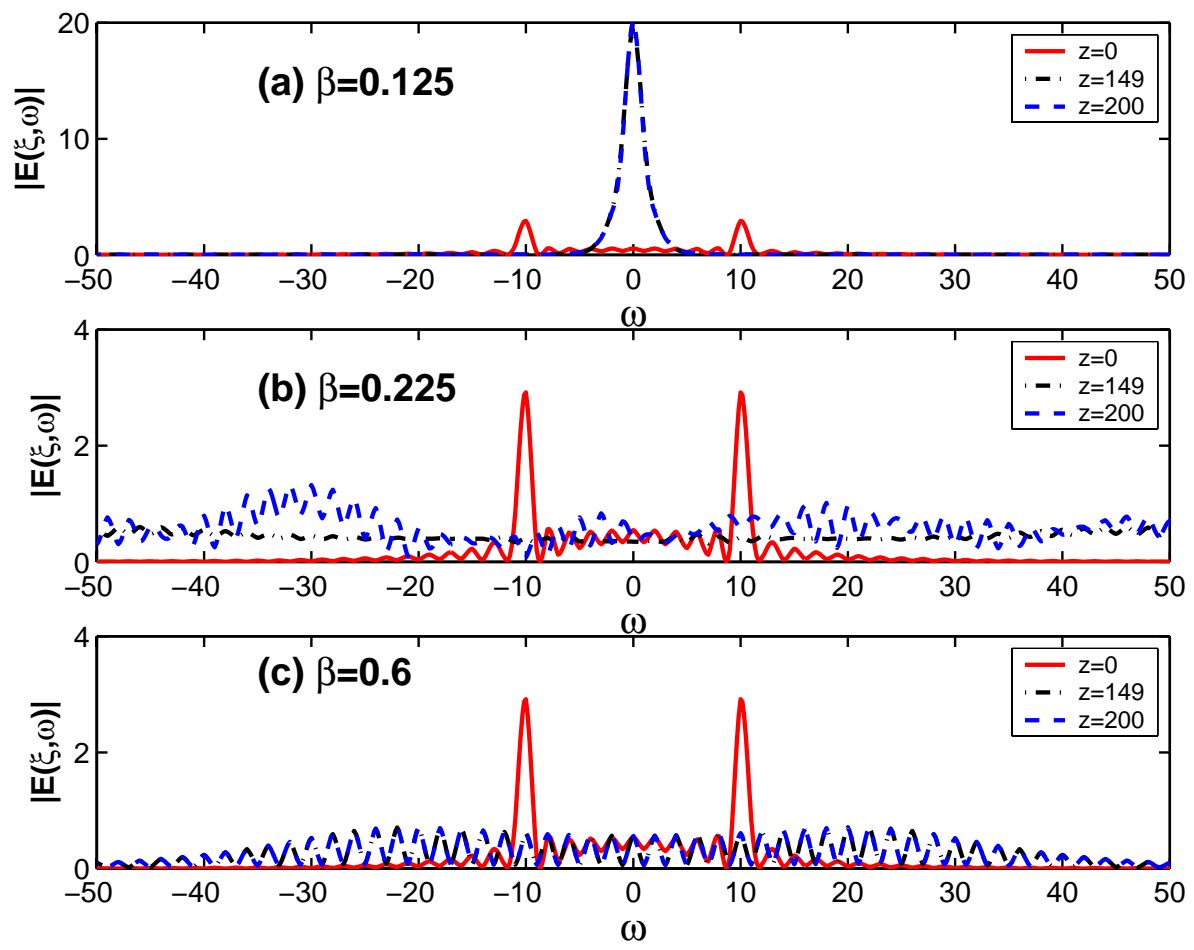

Fig. 6. Plots of the Fourier spectrum amplitudes at different evolution distances of Figures $5 \mathrm{a}-\mathrm{c}$. (a) The initial Fourier spectrum quickly evolves to that of the kink. (b) Asymmetric spectrum broadening and the initial spectrum peaks (solid lines) decay to the weak spectrum amplitude covering the frequencies range. (c) The Fourier spectrum shows asymmetric frequency broadening and the stability indicated by the same spectrum shape at $z=149$ and $z=200$, respectively.
It is shown in Fig. 5a that even in the presence of the modulated field the initial pulse transforms into a front, similar to the case of the $\mathrm{CW}$ under $\mathrm{MI}$ as shown in Fig. 2c, which is also clearly depicted in the Fourier spectrum in Fig. 6a where the initial spectrum peaks transform to the central peak at $z=200$. However, when the gain value slightly increases up to $\beta=0.225$, the initial sech profile under the modulated field results in two erupting solitons: the soliton from the initial pulse at the center and the other from the decay of the 'wall-like' structure induced by the modulated field as shown in Figure 5b. On the other hand, the evolution characteristics from the Fourier spectrum in Fig. $6 \mathrm{~b}$ are quite different from the cases of the pulsating soliton in Figs. $4 \mathrm{a}-\mathrm{c}$ in the senses that it shows an asymmetric spectrum broadening and the initial spectra peaks (solid lines) quickly decay to the weak spectrum amplitude (note that the spectra are normalized to the same scale) covering the frequencies range. For the case of $\beta=0.6$ as shown in Fig. $5 c$, the initial profile transforms into the two solitons with fixed-shape, similar to the cases of Figs. $3 c-d$. The Fourier spectra in Fig. 6c also show asymmetric frequency broadening and the stability indicated by the same spectrum shape at $z=149$ and $z=200$, respectively. From these results, we can conclude that the effects of the gain value $\beta$ are to remove the chaotic nature of the erupting soliton and induce stable fixed-shape multiple solitons.

The effect of the gain term on the normalized energy evolution is shown in Figure 5d. The solid line shows the sudden jump in the energy up to $R_{\varepsilon} \approx 30$ due to the formation of the front as shown in Figure 5a. For the case of $\beta=0.225$, the energy (dot-dashed line) shows the initial eruption as the result of the wall-like structure followed by the subsequent energy burst returning to the initial energy state, i. e., $R_{\varepsilon}=1$. However, for the case of the two fixed-shape solitons, the dashed line indicates their stability by the flat variation of the energy.

\subsection{Effect of the Modulated Field on the Creeping Soliton}

Here, we investigate the effect of a modulated field on the creeping soliton which is formed when an initial rectangular pulse with the two fronts and one sink on the top pulsates back and forth relative to the sink asymmetrically at the two sides of the soliton [6]. We use the same parameters as in Fig. 1c and the modulated field parameters used above. The space-time evolution and the contour plots and the normalized en- 
(a) $\beta=0.101$
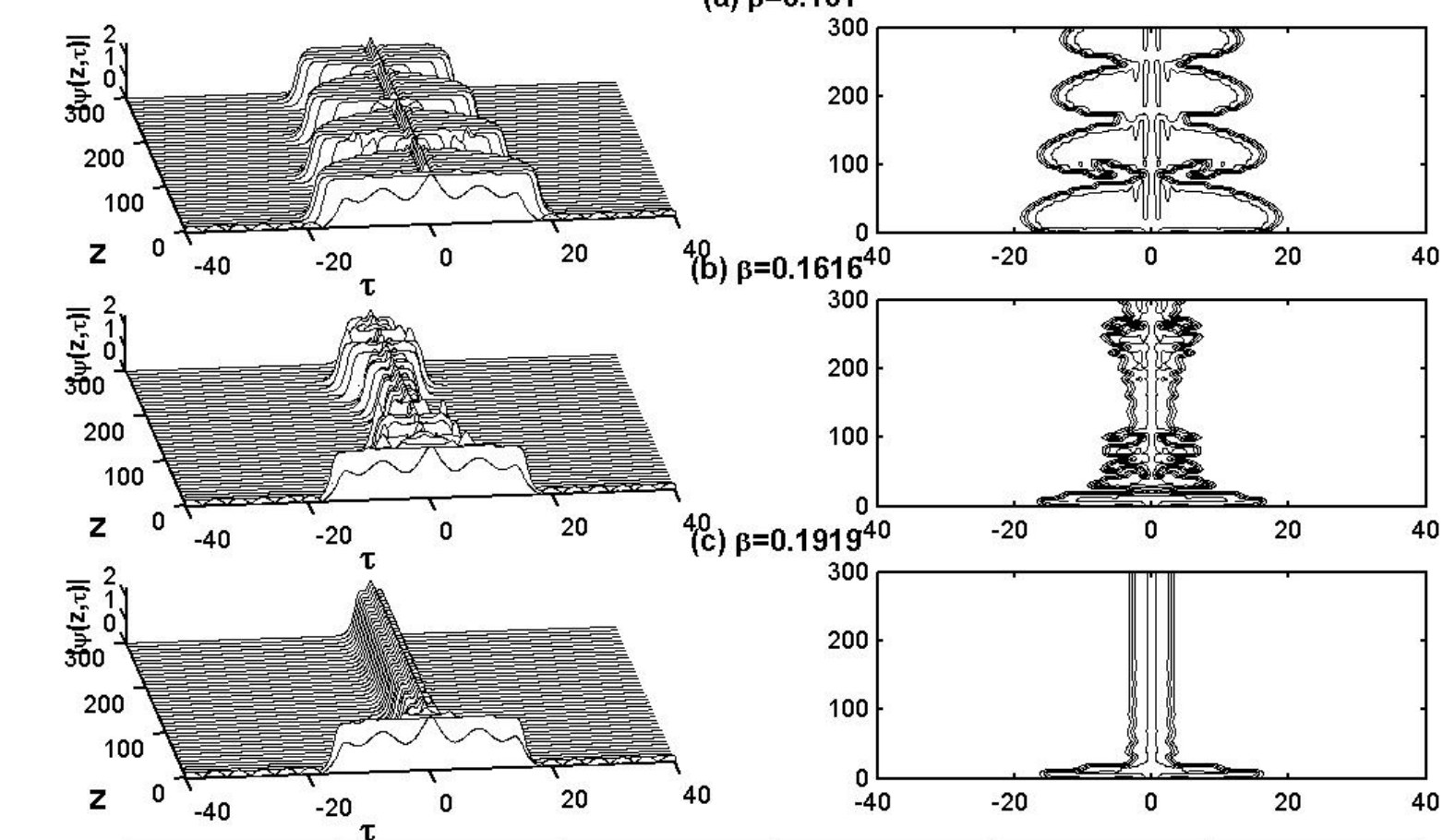

(c) $\beta=0.1919^{40}$
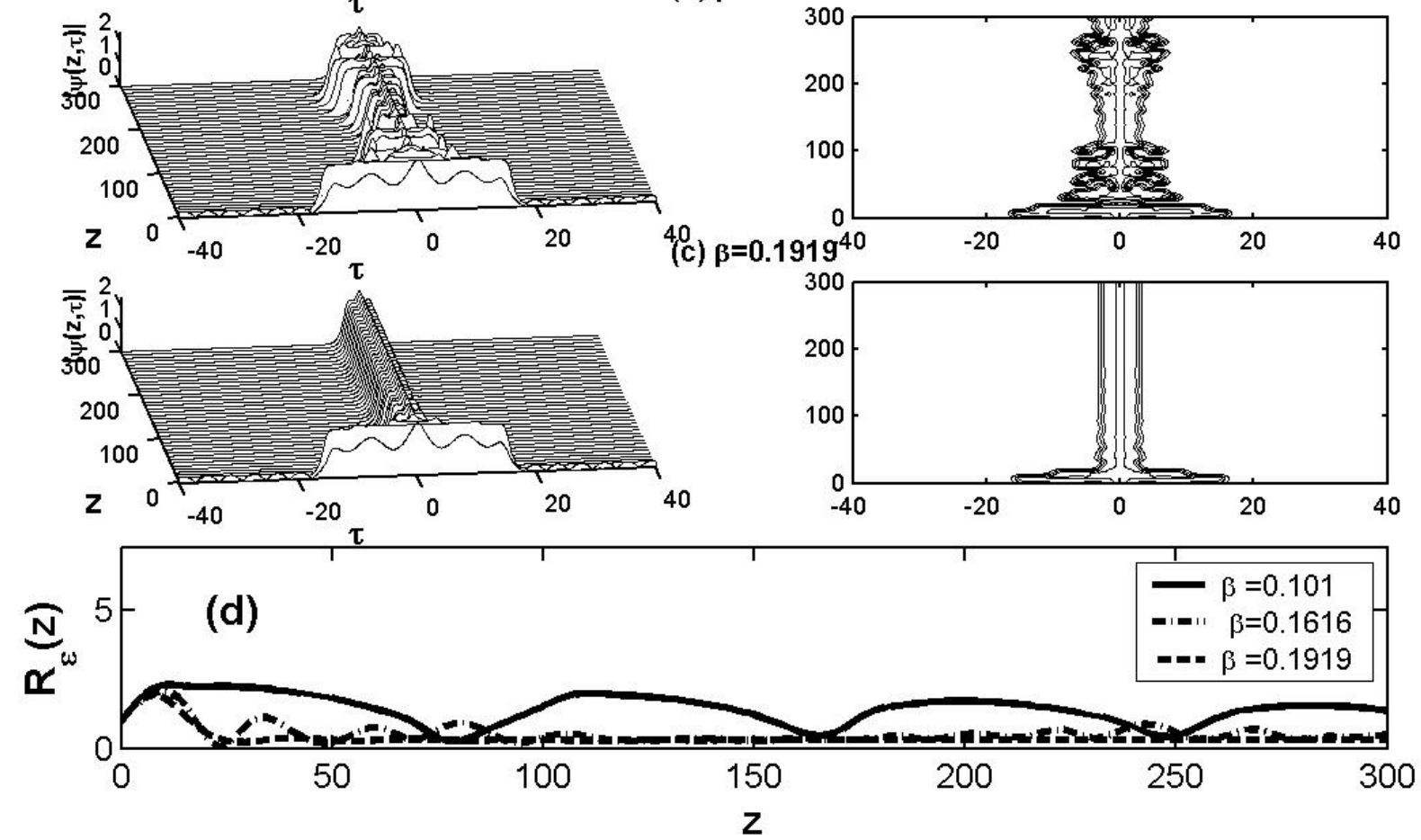

Fig. 7. The space-time evolution and contour plots of the modulus $\psi$ for the parameters of the creeping soliton with different gain values $\beta$. (a) The modulated field alone does not influence the dynamics of the creeping soliton. (b) The perturbed field is suppressed and the width of the creeping soliton varies along the evolution distance. (c) The transformation of the modulated profile to a fixed-shape soliton occurs after the short transient state and subsequent pulsation. (d) The pulsation in the energy evolution disappears as $\beta$ increases.

ergy evolution are calculated in Figs. $7 \mathrm{a}-\mathrm{d}$ for different $\beta$ values.

On the contrary to the previous cases, it should be pointed out that the modulated field alone does not influence the dynamics of the creeping soliton as shown in Fig. 7a since it acts as a strong attractor. It is seen that the modulated field on the left- and right-hand side are suppressed so that the multiple solitons do not appear and only the slightly modified initial profile at the center evolves to the usual creeping soliton in Figure 1d. On the other hand, for the initial Fourier spectrum represented by the solid line in Fig. 8a, the peak at the center with symmetrical oscillatory wings evolves into two peaks at $z=146$ and $z=300$, respectively, as is expected for the creeping soliton due to the pulsation of the two fronts. However, as the gain value increases up to $\beta=0.1616$ in Fig. $7 \mathrm{~b}$, even though the perturbed field is suppressed, the width of the initial profile at the center varies along the evolution distance as clearly depicted in the contour plot, while the creeping nature of the soliton is still maintained. The spectrum snap shots in Fig. $8 \mathrm{~b}$ show that the range of spectrum frequency spreads at $z=146$ and two broad spectrum peaks appear at $z=300$. For a further increased gain value of $\beta=0.1919$, Fig. 7c shows the transformation of the modulated profile to a fixed-shape soliton after a 

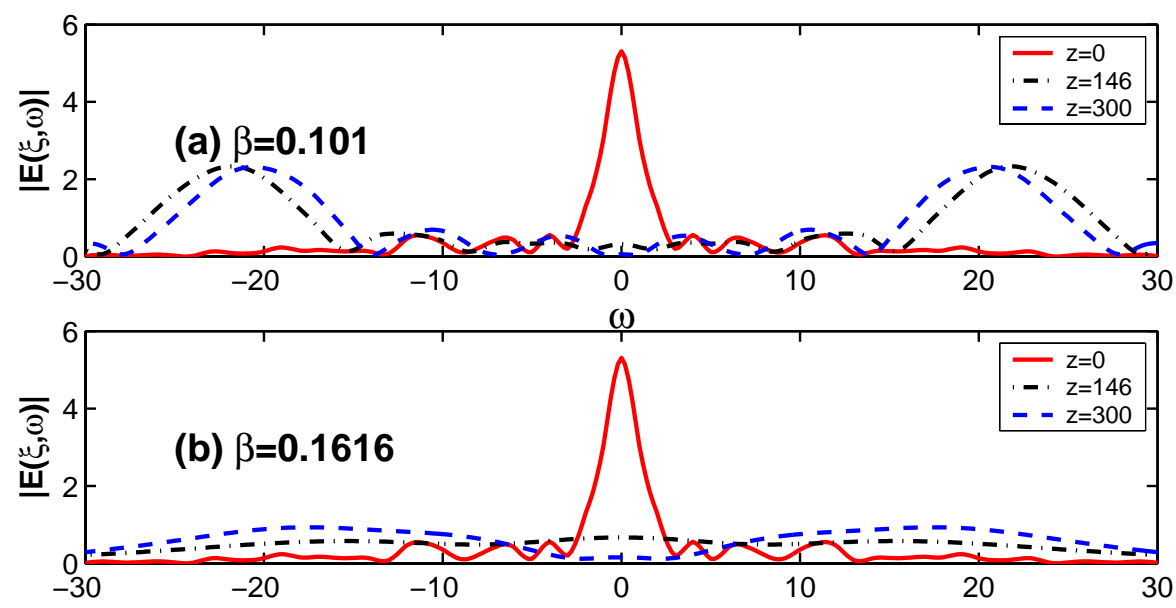

Fig. 8. Plots of the Fourier spectrum amplitudes at different evolution distances of Figures $7 \mathrm{a}-\mathrm{c}$. (a) The initial peak at the center with symmetrical oscillatory wings evolves into two peaks at $z=146$

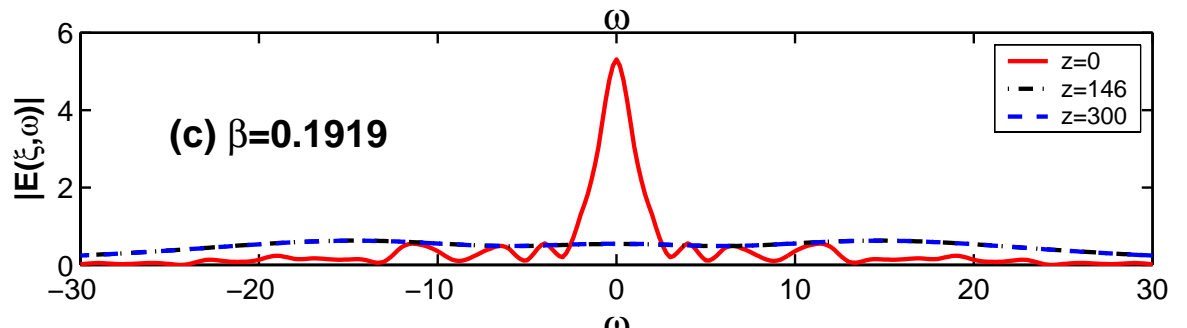
and $z=300$, respectively, due to the pulsation of the two fronts. (b) The range of spectrum frequency spreads at $z=146$ and two broad spectrum peaks appear at $z=300$. (c) The broad frequency range indicates the stability of the soliton.

short transient and subsequent pulsation, of which the stability is indicated by the Fourier spectrum in Fig. 8c due to the broad frequency range. Finally, we note that the characteristic behaviors of the creeping solitons in Fig. 7 can only occur in the presence of both the modulated field and large gain value.

The solid line in Fig. 7d shows a periodic pulsation in the energy evolution similar to that of the creeping soliton in Figure 1d. However, the dot-dashed and dashed lines, respectively, show energy pulsations with gradually decreasing peaks as $\beta$ increases. The dotdashed line demonstrates the stability of the soliton, which shows the flat energy variation after the second peak at $z \approx 60$ where the creeping soliton transforms to the stable breathing soliton.

\section{Conclusions}

In this work, we have investigated the effects of a modulated field and a gain term on the dynamics of the PEC solitons in the context of the cubic-quintic Ginzburg-Landau equation. We have calculated the MI gain function from the linear instability analysis and performed the numerical simulations for the parameters of the PEC solitons and found that the $\mathrm{CW}$ under MI quickly transforms into the fronts as shown in Figure 2. However, it was shown that for the parameters of the pulsating and erupting solitons the pulse-type initial profile added by the modulated field induces the multiple solitons which subsequently transform into stable fixed-shape solitons as the strength of the gain value $\beta$ increases, as demonstrated in Fig. 3 and 5, respectively. The stability of the multiple solitons has been confirmed by the energy evolutions in Fig. 3d and Fig. $5 d$, showing flat variation along the evolution distance as $\beta$ increases. Finally, we have found in Fig. 7 that the presence of the modulated field does not induce the multiple solitons. However, with large $\beta$ values, it was shown that the fixed-shape soliton can be also generated as shown in Figure 7.

\section{Acknowledgements}

This research was supported by the second stage of Brain Korea 21 (BK 21). 
[1] I. S. Aranson and L. Kramer, Rev. Mod. Phys. 74, 99 (2002).

[2] P. Kolodner, Phys. Rev. A 44, 6448 (1991).

[3] M. Dennin, G. Ahlers, and D. S. Cannell, Phys. Rev. Lett. 77, 2475 (1996).

[4] K. G. Müller, Phys. Rev. A 37, 4836 (1988).

[5] Y. Kuramoto, Chemical Oscillations, Waves and Turbulence, Springer, Berlin 1984.

[6] N. Akhmediev and J. M. Soto-Crespo, Phys. Rev. E 70, 036613 (2004).

[7] H. Haus, J. Appl. Phys. 46, 3049 (1975).

[8] J.M. Soto-Crespo, N. N. Akhmediev, and A. Ankiewicz, Phys. Rev. Lett. 85, 2937 (2000).
[9] N. N. Akhmediev, J.M. Soto-Crespo, and G. Town, Phys. Rev. E 63, 056602 (2001).

[10] S. T. Cundiff, J. M. Soto-Crespo, and N. Akhmediev, Phys. Rev. Lett. 88, 073903-1 (2002).

[11] H.P. Tian, Z.H. Li, J.P. Tian, G. S. Zhou, and J. Zi, Appl. Phys. B 78, 199 (2004).

[12] L. Song, L. Lia, Z. Lia, and G. Zhoua, Opt. Commun. 249, 301 (2005).

[13] W. P. Hong, Z. Naturforsch. 61a, 23 (2006).

[14] G. P. Agrawal, Nonlinear Fiber Optics, Optics and Photonics, 3rd ed., Academic Press, New York 2001.

[15] W. P. Hong, Z. Naturforsch. 60a, 719 (2005).

[16] J. M. Soto-Crespo, N. N. Akhmediev, and G. Town, J. Opt. Soc. B 19, 234 (2002). 